A $\mathbf{R}_{\text {EvIEW }}$

\title{
Impact of calorie restriction on epigenetic factors and metabolic pathways during senescence
}

\author{
Upasana, Shaista Parveen and Archana Chakravarty
}

\begin{abstract}
Ageing is an irreversible process associated with numerous physiological alterations across multiple organ systems. It is determined by genetic and epigenetic background in addition to environmental factors associated with social structure, culture and lifestyle. Recently different studies have shown that calorie restriction (CR) plays a noticeable role in the path of delaying senescence by modulating epigenetic factors and metabolic pathways. CR elicits co-ordinated adaptive stress responses at the cellular and whole organism level by modulating epigenetic mechanisms (e.g., DNA methylation, histone modification and miRNA modification ), signaling pathways that regulate cell growth and ageing (e.g., TOR, AMPK, p53 and FOXO ) and cell-to cell signaling molecules (e.g., adiponectin ). Despite of this fact, CR appears to be one of the common ways to increase lifespan in all species. Nutrient sensing pathways are also considered as contributing factors in ageing process because several nutrients can activate different pathways directly or indirectly. Therefore, this review paper focuses on how the epigenetic factors can be influenced by CR during senescence.
\end{abstract}

Key Words : Calorie restriction, Cellular metabolism, Ageing, Epigenetic mechanism, Senescence

How to cite this article : Upasana, Parveen, Shaista and Chakravarty, Archana (2015). Impact of calorie restriction on epigenetic factors and metabolic pathways during senescence. Food Sci. Res. J., 6(1): 148-157. 